\title{
Aspectos hídricos de alguns solos da Amazônia-Regiào do baixo rio Negro
}

\author{
Klaus Reichardt ("); Guido Ranzani ("*); Elias de Freitas Jr. ("*) e Paulo Leonel Libardi (")
}

\section{Resumo}

No presente trabalho, é feito um confronto entre os dados climatológicos, a água disponivel e a condutividade hidráulica saturada de nove perfis de solos da região do baixo rio Negro, no Estado do Amazonas. Os resultados tendem sugerir a presença de uma relativamente baixa capacidade de armazenamento de água disponivel nos perfis de solo estudados. Em média a condutividade hidráulica saturada é representada por $35 \mathrm{~mm} /$ hora, concluindo-se que chuvas com intensidade igual ou maior do que $35 \mathrm{~mm} /$ hora praticamente se constituirão em deflúvio que se perde por escoamento superficial.

\section{INTRODUÇÃo}

A ocupação racional da Amazônia exige um conhecimento profundo dos processos que determinam esse ecossistema. Inúmeros esforços têm sido desenvolvidos com este objetivo nas mais diversas áreas de conhecimento e este trabalho, em particular, procura ampliar a fronteira de nossos conhecimentos em aspectcs referentes às condições hídricas de alguns solos típicos dessa região.

A Amazônia, sendo uma região tropical úmida, é sensivelmente afetada pelo cicio hidrológico. Manaus apresenta, segundo Villa Nova et al. (1976), uma normal de precipitação de $2101 \mathrm{~mm}$ ano-1 e, segundo estes mesmos autores, o fluxo de evapotranspiração médio da bacia como um todo, estimado pela fórmula de Penman, é da ordem de $4 \mathrm{~mm} \mathrm{dia}{ }^{-1}$, correspondendo a $61,8 \%$ do balanço hídrico. Estes dados, quando analisados em relação aos diferentes solos da regiăo, evidenciam proble mas de escoamento superficial de água e de lixiviação dos perfís. Estes problemas neces sitam forçosamente de ser levados em consideração quando da substituição da vegetação nativa pelos diversos sistemas de produção agropecuária. No presente trabalho, é feito um confronto entre dados climatológicos existentes e algumas propriedades físicas de nove perfís típicos de solos da região de Manaus, discutindo os aspectos hídricos de cada perfil.

\section{MATERIAIS E MÉTODOS}

Os solos utilizados no presente estudo pertencem à Estação Experimental de Silvicultura Tropical do INPA, situada no $\mathrm{Km} 45$ da BR 174 que liga Manaus ( $A M)$ a Caracarai $(R O)$. Os resultados dos exames morfológicos são apresentados por Ranzani (1979) .

Com as amostras de cada horizonte, procedeu-se à determinação da água disponivel. utilizando sua definição clássica. Os autores estão cientes das limitações deste conceito e o utilizam apenas para confronto relativo entre solos e para possibilitar sua comparação com perfís de outras regiões do Brasil.

Os teores de água de amostras de TFSA submetidas a potenciais de pressão de $1 / 3$ e 15 atmosferas foram obtidos em placas porosas, segundo Richards (1965) .

A água disponível (AD) de cada horizonte foi calculada pela expressão:

$$
A D(m m)=\frac{\left[\mathrm{U}_{1 / 3}-\mathrm{U}_{15}\right] \mathrm{Da}}{10} \times \mathrm{L} \ldots .
$$

onde $U_{1} / 3$ e $U_{15}$ são as umidades \% peso de amostras submetidas a potenciais de pressão de $1 / 3$ e 15 atmosferas, respectivamente; Da a densidade aparente $\left(\mathrm{g} . \mathrm{cm}^{-3}\right)$ do horizonte e $\mathrm{L}(\mathrm{cm})$ a espessura do horizonte .

A condutividade hidráulica saturada Ko $\left(\mathrm{cm} \cdot \mathrm{h}^{-1}\right)$ foi determinada em laboratório, segundo o método proposto por Klute (1965), compactando as amostras até uma densidade

(') - Centro de Energia Nuclear na Agricuitura, Piracicaba, SP (*) - Instituto Nacional de Pesquisas da Amazônia, Manaus. 
aparente idêntica à encontrada no campo. A fig. 1 mostra um esquema do permeâmetro utilizado.

A condutividade Ko foi calculada pela expressão :

$$
K_{\mathrm{o}}=\frac{\mathrm{g} \mathrm{L}}{\mathrm{h}+\mathrm{L}} \ldots \ldots \ldots \ldots \ldots \ldots \ldots \ldots .
$$

onde q é o fluxo de água $\left(\mathrm{cm}^{3} \cdot \mathrm{cm}^{-2} \cdot \mathrm{h}^{-1}\right)$ necessário para manter o nível de água $\mathrm{h}$. $(\mathrm{cm})$ constante; sendo $\mathrm{L}$ a espessura da camada de terra.

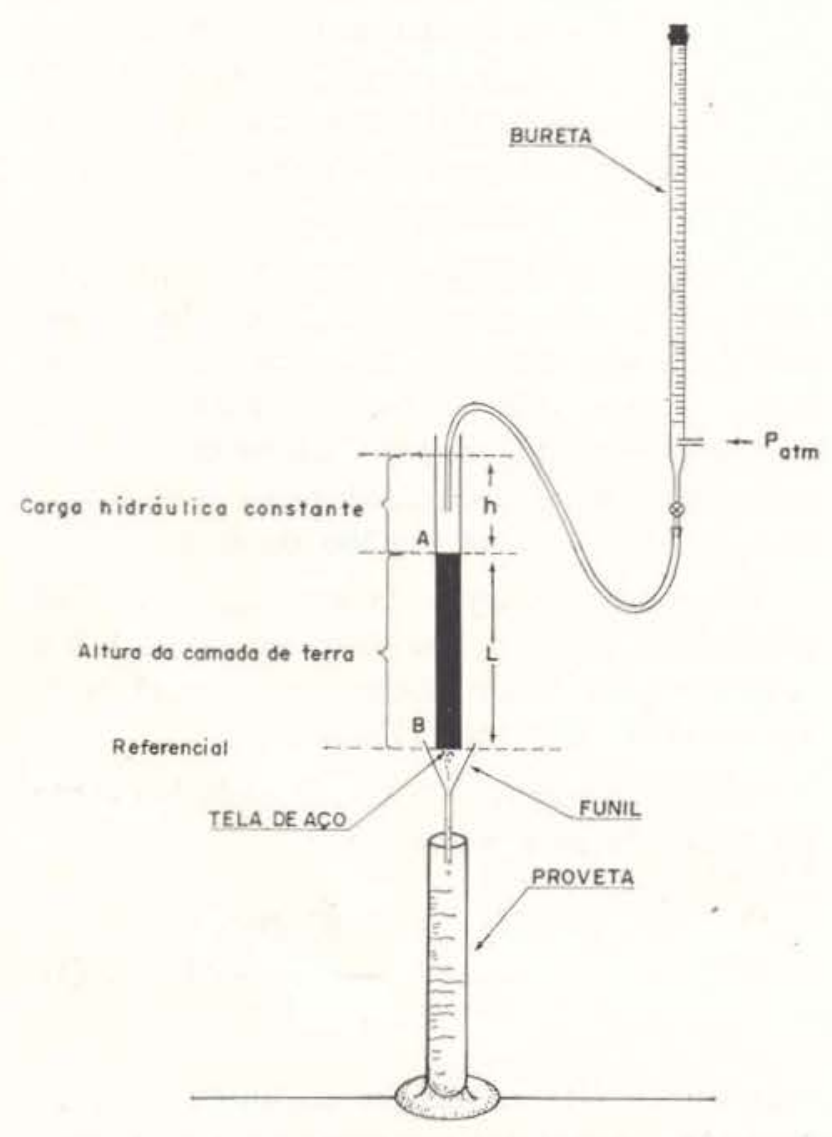

Fig. 1 - Diagrama do sistema de carga hidráulica constante para medida da condutividade hidráulica saturada.

\section{Resultados}

O quadro 1 apresenta os valores de $\mathrm{U}_{1 / 3}$ e U15 para os diferentes horizontes dos perfís amostrados até a profundidade de $100 \mathrm{~cm}$, considerados neste estudo. Com estes dados. calcularam-se os valores de $A D$, utilizando a expressão (1) e empregando valores de $\mathrm{L}=$ 30,60 e $100 \mathrm{~cm}$ de profundidade.

Estes valores foram escolhidos porque a camada da risosfera da maioria das culturas agrícolas oscila entre 30 e $60 \mathrm{~cm}$ de profundidade, podendo, às vezes, alcançar $100 \mathrm{~cm}$. Os dados de $A D$ e da condutividade hidráulica saturada, $\mathrm{K}_{\mathrm{o}}$, dos horizontes superficiais de cada perfil, encontram-se no Quadro 2.

\section{QUADRO 1}

\begin{tabular}{|c|c|c|c|c|c|}
\hline Perfil & $\begin{array}{c}\text { Hori- } \\
\text { zonte }\end{array}$ & $\begin{array}{c}\text { Espessura } \\
\text { (cm) }\end{array}$ & $\begin{array}{c}\mathrm{Da} \\
\left(\mathrm{g} . \mathrm{cm} \cdot{ }^{-3}\right)\end{array}$ & $\begin{array}{c}U_{1 / 3} \\
\text { (\% peso) }\end{array}$ & $\begin{array}{c}u_{15} \\
\text { (\% peso) }\end{array}$ \\
\hline \multirow[t]{4}{*}{1} & $A_{11}$ & $0-25$ & 1.40 & 13.0 & 10.2 \\
\hline & $A_{12}$ & $25-60$ & 1.36 & 5.2 & 2.9 \\
\hline & $\mathrm{A}_{3}$ & $60-88$ & 1.47 & 13.47 & 9.0 \\
\hline & $\mathrm{B}_{21}$ & $88-120$ & 1.52 & 12.5 & 8.6 \\
\hline \multirow[t]{4}{*}{2} & $A_{11}$ & $0-17$ & 1.36 & 11.6 & 8.1 \\
\hline & $A_{12}$ & $17-45$ & 1.34 & 17.9 & 12.9 \\
\hline & $\mathrm{A}_{3} / \mathrm{B}_{1}$ & $45-72$ & 1.38 & 18.5 & 14.7 \\
\hline & $\mathrm{B}_{21}$ & $72-122$ & 1.51 & 18.7 & 16.0 \\
\hline \multirow[t]{4}{*}{3} & $A_{11}$ & $0-12$ & 1.29 & 29.6 & 21.6 \\
\hline & $A_{12}$ & $12-44$ & 1.33 & 30.3 & 25.1 \\
\hline & $A_{3} / B_{1}$ & $44-60$ & 1.31 & 31.6 & 26.2 \\
\hline & $\mathrm{B}_{21}$ & $60-115$ & 1.20 & 32.2 & 27.5 \\
\hline \multirow[t]{4}{*}{4} & $A_{1}$ & $0-15$ & 1.54 & 18.5 & 12.1 \\
\hline & $\mathrm{A}_{3}$ & $15-40$ & 1.30 & 25.5 & 17.8 \\
\hline & $B_{21}$ & $40-68$ & 1.37 & 32.2 & 22.4 \\
\hline & $\mathrm{B}_{22}$ & $68-120$ & 1.30 & 37.5 & 28.3 \\
\hline \multirow[t]{5}{*}{5} & $A_{1}$ & $0-17$ & 1.50 & 15.6 & 10.5 \\
\hline & $\mathrm{A}_{3}$ & $17-48$ & 1.30 & 25.1 & 18.2 \\
\hline & $\mathrm{B}_{21}$ & $48-62$ & 1.38 & 27.2 & 21.4 \\
\hline & $\mathrm{B}_{22}$ & $62-95$ & 1.30 & 29.1 & 23.4 \\
\hline & $\mathrm{B}_{3}$ & $95-126$ & 1.24 & 25.6 & 23.1 \\
\hline \multirow[t]{2}{*}{6} & $A_{11}$ & $0-12$ & 1.40 & 7.4 & 6.2 \\
\hline & $A_{12}$ & $12-31$ & 1.40 & 10.7 & 8.6 \\
\hline & $A_{13}$ & $31-66$ & 1.41 & 12.3 & 10.9 \\
\hline & $A_{3} / B_{1}$ & $66-95$ & 1.42 & 12.6 & 10.0 \\
\hline & $\mathrm{B}_{12}$ & $95-136$ & 1.41 & 12.4 & 10.7 \\
\hline \multirow[t]{4}{*}{7} & $A_{11}$ & $0-18$ & 1.20 & 35.4 & 28.2 \\
\hline & $A_{12}$ & $18-47$ & 1.20 & 37.8 & 30.1 \\
\hline & $B_{21}$ & $47-91$ & 1.07 & 40.3 & 32.5 \\
\hline & $\mathrm{B}_{22}$ & $91-133$ & 1.12 & 40.8 & 35.0 \\
\hline \multirow[t]{4}{*}{8} & $A_{11}^{2}$ & $0-14$ & 1.17 & 34.8 & 29.2 \\
\hline & $A_{12}$ & $14-44$ & 1.26 & 37.1 & 31.8 \\
\hline & $\mathrm{A}_{3} / \mathrm{B}_{1}$ & $44-85$ & 1.09 & 39.0 & 32.8 \\
\hline & $\mathrm{B}_{21}$ & $85-147$ & 1.05 & 41.9 & 35.0 \\
\hline \multirow[t]{4}{*}{9} & $A_{11}$ & $0-12$ & 1.16 & 38.4 & 33.0 \\
\hline & $A_{12}$ & $12-39$ & 1.22 & 35.9 & 32.2 \\
\hline & $\mathrm{A}_{3} / \mathrm{B}_{1}$ & $39-72$ & 1.17 & 36.6 & 32.7 \\
\hline & $\mathrm{B}_{21}$ & $72-115$ & 1.45 & 37.1 & 33.8 \\
\hline
\end{tabular}


QUADRO 2

\begin{tabular}{|c|c|c|c|c|}
\hline Perfil & $\begin{array}{l}\mathrm{AD}_{30} \\
(\mathrm{~mm})\end{array}$ & $\begin{array}{l}A D_{60} \\
(\mathrm{~mm})\end{array}$ & $\begin{array}{l}\mathrm{AD}_{100} \\
(\mathrm{~mm})\end{array}$ & $\underset{K_{0}}{\left(m m . h .^{-1}\right)}$ \\
\hline 1 & 11,4 & 20.9 & 47,6 & 22,7 \\
\hline 2 & 16,9 & 34.8 & 52,2 & 99,7 \\
\hline 3 & 24.8 & 45.4 & 67,8 & 10,2 \\
\hline 4 & 30,1 & 67,0 & 116,1 & 33,2 \\
\hline 5 & 24,7 & 50.7 & 80,5 & 18.1 \\
\hline 6 & 7,4 & 13,2 & 26,3 & 74,8 \\
\hline 7 & 26,9 & 53,5 & 85,1 & 25,2 \\
\hline 8 & 19,7 & 39,6 & 67,1 & 26,3 \\
\hline 9 & 15,7 & 29,5 & 48,4 & 10,1 \\
\hline Médias & 19.7 & 39,4 & 65,7 & 35,6 \\
\hline
\end{tabular}

\section{DISCUSSÃo}

Os dados obtidos mostram, de uma maneira geral, a baixa capacidade de retençăo de água dos perfís estudados. Com um fluxo de evapotranspiração da ordem de $4 \mathrm{~mm} \mathrm{dia}^{-1}$, como mostram Villa Nova et al. (1976), a água retida pela camada $0-30 \mathrm{~cm}$ seria perdida em praticamente 2 a 7 dias para os solos estudados; na camada de $0-60 \mathrm{~cm}$, a água retida seria perdida em 3 a 17 dias, enquanto na camada de $1 \mathrm{~m}$, em 7 a 29 dias.

Villa Nova et al. (1976) apresentam valores médios de precipitação mensal obtidos no período 1931-1960 na Estação de Manaus :

J $F \quad M \quad A \quad M \quad J \quad J \quad A \quad S \quad O \quad N \quad D \quad A n$ 27627730128719398614162112165228210

O problema da baixa retenção de água aliado às características das chuvas da região, que em geral são intensas e de pequena auração, o que não é mostrado no quadro acima. tendem a restringir as percolações profundas em favor do deflúvio ou escoamento superficial.

Ribeiro (1976) também apresenta dados detalhados sobre o clima da região de Manaus. Segundo este autor, um balanço hídrico feito segundo Thorntwaite \& Matter (1955), apresentou um excesso de $970 \mathrm{~mm}$ e um déficit de $28 \mathrm{~mm}$. Este mesmo autor apresenta ainda dados de intensidade máxima de chuvas, podendo estas alcançai cifras acima de 100 $\mathrm{mm} \cdot \mathrm{h}^{-1}$.

Confrontando os dados apresentados no Quadro $2 \mathrm{com}$ estes dados climáticos, pode-se concluir (em termos médios) que chuvas de intensidade maior que $35 \mathrm{~mm} . \mathrm{h}^{-1}$, forçosamente apresentarão intenso escoamento superficial. Chuvas de intensidade menor podem também trazer problemas de escoamento superficial devidos à baixa capacidade de retenção de água dos perfís. Cerca de $20 \mathrm{~mm}$ são suficientes para completar a camada $0-30 \mathrm{~cm}$. se esta estiver completamente seca. É difícil fazer estimativas das percentagens de água armazenada no solo, escoada pela superfície ou percolada, de vez que estes processos dependem de condições locais, cobertura do solo, declividade do terreno, umidade do solo no momento da chuva, etc. Pode dizer-se que em termos médios chuvas de mais de $20 \mathrm{~mm}$ completarão a camada $0-30 \mathrm{~cm}$, havendo mais escoamento superficial do que percolação para horizontes mais profundos, em virtude da intensidade das chuvas que, em geral, é elevada. A freqüência com que o escoamento superficial incide deve agravar os efeitos da erosão. como mencionado por outros autores, na região (Ranzani, 1979) .

Os resultados sugerem a necessidade de condução de trabalhos nos quais sejam feitas medidas diretas dos componentes hídricos para uma avaliação mais precisa das condições oferecidas pelo solo às plantas.

\section{SUMMARY}

Some relationship between climatological data, available water and saturated hydraulic conductivity of nine representative soil profiles of the low rio Negro, Amazon State region, were studied.

The results shows a relatively low available water retention of all soils studied. A saturated hydraulic conductivity of $35 \mathrm{~mm} \cdot \mathrm{h}^{-1}$ was the mean value obtained. This means that rains with the intensity of $35 \mathrm{~mm} \cdot \mathrm{h}^{-1}$ or more, will be converted almost exclusively into run-off water. 


\section{BIBLIOGRAFIA}

KLUTE, A.

1965 - Laboratory Measurement of Hydraulic Conductivity of Saturated Soil. In: Black, C.A. Methods of Soil Analysis. Part I. American Society of Agronomy, Inc. Publisher, Medison, Wisconsin, USA. p 210-221.

RANZANI, G.

- Identificação e Caracterização de Alguns Solos da Estação Experimental de Silvicultura Tropical do INPA. Acta Amazonica, 10(1) (neste volume).

RibeIRo, M.N.G.

1976 - Aspectos Climatológicos de Manaus. Acta Amazonica, 6(2) : 229-234.
RICHARDS, L.A.

1965 - Physical Condition of Water in Soil. In: Black, C.A. ed - Methods of Soil Analysis. Part. 1. American Society of Agronomy, Inc. Publisher, Medison, Wisconsin, USA. p. 128-151.

Thorntwaite, C.W. \& MATHER, J.R.

1955 - The water balance. Public. in Climatology. (1). Centerton, N.Y.

Villa nova, N.A.; Salati, E. \& Matsui, E.

1976 - Estimativa da Evapotranspiração na Bacia Amazônica. Acta Amazonica, 6(2) : 215-228.

(Aceito para publicação em 15/10/79). 